Europhys. Lett., 70 (4), pp. 432-438 (2005)

DOI: $10.1209 / \mathrm{epl} / \mathrm{i} 2005-10021-7$

\title{
Characterization of stochastic resonance
}

\author{
K. PARK ${ }^{1}$ and Y.-C. LAI ${ }^{2}$ \\ 1 Department of Mathematics, Arizona State University - Tempe, AZ 85287, USA \\ 2 Departments of Electrical Engineering and Physics, Arizona State University \\ Tempe, AZ 85287, USA
}

received 3 February 2005; accepted in final form 31 March 2005

published online 20 April 2005

PACS. 05.40.-a - Fluctuation phenomena, random processes, noise, and Brownian motion. PACS. 02.50.Ey - Stochastic processes.

\begin{abstract}
Traditional quantities used to characterize stochastic resonance possess the common feature of low sensitivity to noise variation in the sense that they vary slowly about the optimal noise level. In potential applications of stochastic resonance such as device development, a high sensitivity to noise may be required. Here we show that, when the resonance is understood as a manifestation of phase synchronization, the average synchronization time between the input and output signal has an extremely high sensitivity in that it exhibits a cusp-like behavior about the optimal noise level. Theoretical analysis and numerical evidence are provided to establish the cusp-like behavior and its generality.
\end{abstract}

The important nonlinear phenomenon of stochastic resonance (SR), since its discovery [1], has stimulated a large amount of research [2-6]. This phenomenon has been identified in a variety of natural and man-made systems, including bistable lasers [7], biological cells [8], and neural systems [9]. Given a nonlinear system, its response to a weak signal is generally influenced by noise but, when SR occurs, noise can enhance the response. To characterize SR, one usually uses signal-to-noise ratio (SNR) [2], correlations [5], entropies and other quantities derived from the information theory [6], and measures how these quantities change as the noise amplitude is increased from zero. Typically, one finds that they can be maximized for an optimal value or for a range of values of the noise amplitude. One common feature associated with these quantities is that they vary smoothly with noise about the optimal value, exhibiting a "bell-shape" behavior. For potential applications such as assessing the working environment based on the principle of SR [10], one might be interested in measures that are sensitive to noise variation. However, a quantity with a "bell-shape" dependence on noise amplitude cannot have a high sensitivity. The purpose of this letter is to present our finding of a measure of SR that has an extremely high sensitivity in the sense that it exhibits, as a function of the noise amplitude, a cusp-like behavior about the optimal noise level. The measure is the average phase-synchronization time. Here we utilize the term "cusp-like" to merely indicate a high sensitivity to noise. It is not rigorous in the sense that our heuristic theory only predicts a fast rising and a fast falling behavior in the average synchronization time when the noise level approaches and becomes larger than the optimal value, respectively. 
Recently, it has been shown that SR can be understood as a manifestation of phase synchronization (PS) between the input and output signal. In particular, the relationship between SR and PS has been demonstrated in noisy bistable systems with periodic and aperiodic signals $[3,4]$ and in excitable systems with periodic [11] and aperiodic signals [12]. To define PS, imagine an input signal $x_{i n}(t)$ that oscillates in time. A phase variable $\phi_{i n}(t)$ can be defined where one cycle of oscillation in $x_{i n}(t)$ corresponds to an increase of $2 \pi$ in $\phi_{i n}(t)$. A similar phase variable $\phi_{\text {out }}(t)$ can be defined for the output signal $x_{\text {out }}(t)$. PS is said to occur [13] if the phase difference satisfies $\Delta \phi(t) \equiv\left|\phi_{\text {out }}(t)-\phi_{\text {in }}(t)\right| \leq 2 \pi$ for all $t$. Clearly, for a noisy nonlinear system such perfect synchronization is not possible. However, PS can occur in finite time intervals in the sense that $\Delta \phi(t)$ can remain bounded within $2 \pi$ for a finite amount of time before a phase slip, typically $2 \pi$, occurs. Given a noise amplitude $D$, one can then measure the average time $\tau(D)$ for phase synchronization. It was found [12] that for an SR system, this time also exhibits a resonant behavior in that it increases with noise, reaches a maximum, and then decreases as the noise level is further raised, as exemplified in fig. 1 for a system of an array of coupled FitzHugh-Nagumo (FHN) oscillators, the paradigmatic model for studying SR [5]. The interesting feature is that $\tau(D)$ exhibits extremely rapid rising and falling behaviors for noise amplitude about the optimal value. More detailed and finer numerical results with the coupled FHN system are difficult because of the demanding computations.

Characterization of SR by using the phase-synchronization time is of fundamental interest because this represents an alternative way to study SR. More importantly, this way of studying SR can be practically useful because the synchronization time depends on the noise level much more sensitively than the traditional measures such as SNR. Suppose an instrument is to be built based on the phenomenon of SR. Then utilizing this time measure can be more advantageous because of the higher precision it can potentially offer. In this letter, we will

(a)

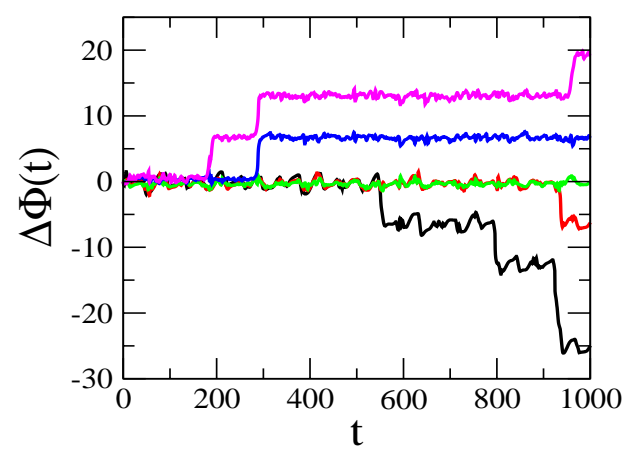

(b)

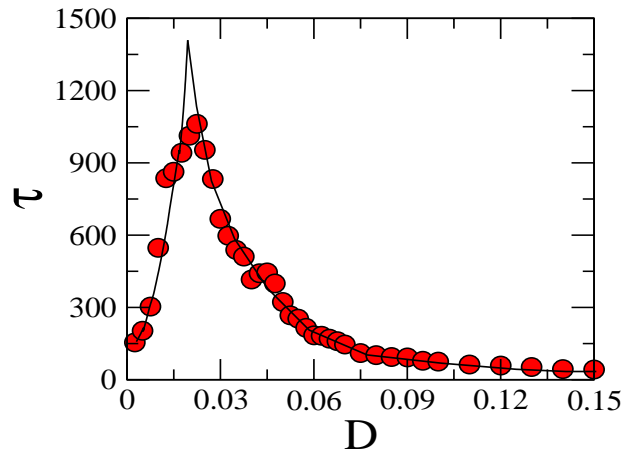

Fig. 1 - (a) Evolutions of the phase difference $\Delta \phi(t)$ between the input and output signal and (b) average phase-synchronization time $\tau$ vs. noise amplitude for an array of 3 FitzHugh-Nagumo (FHN) oscillators [14] given by $0.005 \dot{x}_{i}=x_{i}\left(x_{i}-1 / 2\right)\left(1-x_{i}\right)-y_{i}+S(t)+0.0015 \eta(t)$, and $\dot{y}_{i}=x_{i}-y_{i}-0.15+D \xi_{i}(t)$, $i=1,2,3$, where $S(t)=0.06 \sin \left[\omega_{0} t+0.5 \cos \left(\omega_{1} t\right)\right]$ is a frequency-modulated input signal, $0.0015 \eta(t)$ is Gaussian white noise associated with the input, and $D \xi_{i}(t)$ are independent, Gaussian white noise to each oscillator used to induce SR. The output of the system is the firing rate associated with the ensemble-averaged spike train $X(t)=\sum x_{i}(t)$, and the corresponding phase variable is calculated by using the standard Hilbert-transform method [13]. In (a), the noise amplitudes for the five traces are $D=0.065,0.045,0.025,0.015$, and 0.005 , respectively (from top to bottom). In (b), for each value of the noise amplitude, 100 realizations were used to obtain the value of $\tau$. Near the optimal noise amplitude, $\tau$ apparently exhibits a cusp-like behavior. 

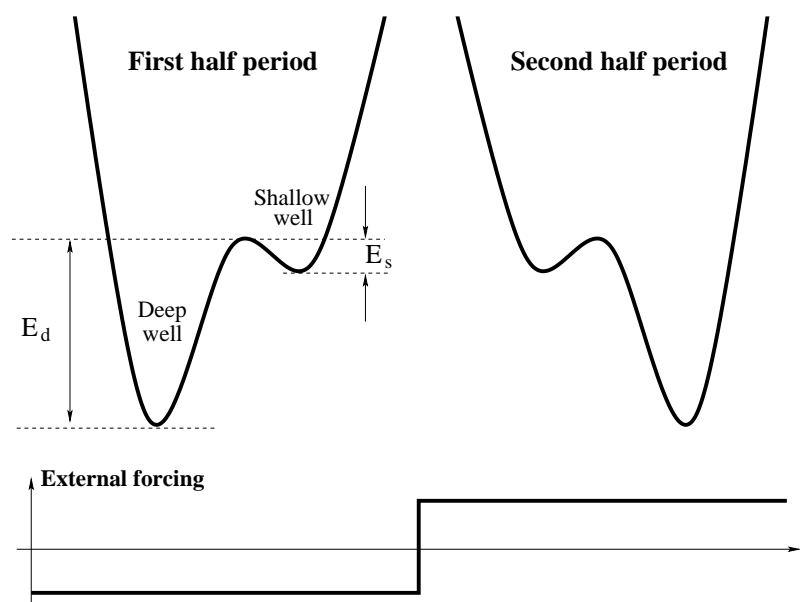

Fig. 2 - Tilted double-well potential as a result of external forcing.

provide a basic understanding of the behavior of the phase-synchronization time associated with SR. To be able to obtain analytic understanding, we utilize the standard double-well potential model with a periodic input signal. The dynamics of phase synchronization is then analyzed based on the transitions between the potential wells, with the help of the Kramers' formula. We find that, near the optimal noise level, the function $\tau(D)$ exhibits a cusplike behavior with distinct values of derivative depending on whether the optimal level is approached from below or above. Although the specifics of $\tau(D)$ depend on the details of the system and input signal, our analysis and numerical computations indicate that the cusp-like behavior is general. While there is a huge body of literature on SR, to our knowledge, the cusp-like behavior in the synchronization time presented here has not been noticed previously.

To provide a theoretical explanation for the cusp-like behavior in the transient synchronization time, we consider the paradigmatic model for stochastic resonance: particle motion in a double-well potential in the presence of external driving and noise, subject to strong damping. The Langevin equation can be written as $\mathrm{d} x / \mathrm{d} t=-\mathrm{d} U(x) / \mathrm{d} x+F(t)+\sqrt{2 D} \xi(t)$, where $U(x)=-x^{2} / 2+x^{4} / 4, D$ is the noise amplitude, and $\xi(t)$ is the white noise term that satisfies $\langle\xi(t)\rangle=0$ and $\left\langle\xi(t) \xi\left(t^{\prime}\right)\right\rangle=\delta\left(t-t^{\prime}\right)$. The potential has two wells at $x_{l}=-1$ and $x_{r}=1$, respectively, and a barrier at $x=0$. For simplicity, we consider the case where the external driving $F(t)$ is a periodic rectangular signal of period $T_{0}=1: F(t)=-F_{0}$ for $0 \leq t<1 / 2$ and $F(t)=F_{0}$ for $1 / 2 \leq t<1$. Letting $V(x, t)=U(x)-F(t) x$ be the effective potential, the Langevin equation becomes $\mathrm{d} x / \mathrm{d} t=-\partial V(x, t) / \partial x+\sqrt{2 D} \xi(t)$. The tilted potential $V(x, t)$ can assume one of two forms in the first and second half of a period, as shown schematically in figs. 2 left and right, respectively. Due to the external forcing, the two wells become asymmetric with respect to each other. At a given time, one of the wells is deep and the other is shallow, and they alternate periodically in time with period $T_{0}$. It is thus natural to assign a phase variable $\phi(t)$ for a particle in a well: $\phi(t)=0$ if the particle is in the well at $x_{r}$ and $\phi(t)=\pi$ if it is in the well at $x_{l}$. Due to noise, a particle initially in one well can overcome the potential barrier to go to the other well, vice versa. The rate of this transition is determined by the Kramers formula [15]: $K \sim \exp \left[-E_{b} / D\right]$, where $E_{b}$ is the barrier height. Let $E_{d}$ and $E_{s}$ be the barrier heights when the particle is in the deep and shallow well, respectively, where $E_{d}>E_{s}$. The rates of transition (the probabilities of transition per unit time) from the deep to the shallow and the opposite are $K_{d} \sim \exp \left[-E_{d} / D\right]$ 
and $K_{s} \sim \exp \left[-E_{s} / D\right]$, respectively. For different noise strength, the response of the particle to the external forcing, as measured by the transitions between the two wells, determines the extent of phase synchronization between the input and output.

Imagine that for some noise strength, in one period of time the probability of transition from the shallow to the deep well is appreciable, but that of the opposite transition is negligible. Assume initially a particle is in the shallow well. Thus, during the first half period the particle moves to the deep well, generating a $\pi$ phase change. In the second half period, the deep well becomes shallow and vice versa and, hence, the particle moves to the shallow well, which is the well that it was initially in, as shown in fig. 2 . There is then another $\pi$ phase change in the second half period. The total phase change in one period is then $2 \pi$, which matches exactly the phase change associated with the input signal. That is, the phase of the particle can be locked with respect to that of the input signal, giving rise to perfect phase synchronization. For realistic system and noise, such a perfect synchronization cannot be achieved. Let $D_{\text {opt }}$ be the noise amplitude for which the average synchronization time reaches a maximum value $\tau_{\max } \gg 1$. Let $P_{2 \pi}(D)$ be the probability for a $2 \pi$ change in one driving period. We thus have $P_{2 \pi}\left(D_{\text {opt }}\right) \approx 1 / \tau_{\max } \equiv \varepsilon$.

Consider first the case $D<D_{\text {opt }}$. In the extreme case where $D$ is slightly greater than 0 , the Kramers rates are essentially zero, so that a particle initially in one potential well will remain there for long time. Due to the $2 \pi$ phase change in the input signal in one period, there will be a corresponding $2 \pi$ change in the phase difference $\Delta \Phi$ between the input and the output signal. We have $P_{2 \pi}(0) \approx 1$. As $D$ is increased from zero, it becomes possible for a particle in the shallow well to hop to the deep well so that $K_{s}$ will increase, but if $D$ is small, we expect $K_{d}$ to remain negligible because of the higher potential barrier. This will reduce $P_{2 \pi}(D)$ from unity. The amount of reduction is given by the Kramers rate $K_{s}$. The probability for $2 \pi$ phase change is thus $\left(1-C_{0} K_{s}\right)$, where $C_{0}$ is a constant that can be determined by the condition $P_{2 \pi}\left(D_{\text {opt }}\right) \approx \varepsilon$. We obtain $P_{2 \pi}^{<}(D)=1-(1-\varepsilon) \exp \left[E_{s} / D_{\text {opt }}\right] \exp \left[-E_{s} / D\right]$. The average phase synchronization time for $D<D_{\text {opt }}$ is thus given by $\tau^{<}(D) \approx 1 / P_{2 \pi}^{<}(D)$. We have

$$
\mathrm{d} \tau^{<}(D) /\left.\mathrm{d} D\right|_{D \rightarrow D_{o p t}^{-}} \approx \frac{(1-\varepsilon) E_{s}}{\varepsilon^{2} D_{o p t}^{2}} .
$$

We see that $\mathrm{d} \tau^{<}(D) /\left.\mathrm{d} D\right|_{D \rightarrow D_{\text {opt }}^{-}} \rightarrow \infty$ for $\epsilon \rightarrow 0$. For small value of $\varepsilon$, we thus expect to observe that $\tau^{<}(D)$ increases rapidly as $D \rightarrow D_{\text {opt }}$ from below.

For $D>D_{o p t}$, it is possible for a particle in the deep well to overcome the high potential barrier to hop to the shallow well. Thus, both transition rates become important. Because of the large noise, in one driving period a particle in the deep well can hop to the shallow well and then moves rapidly to the deep well again because of the relatively low potential barrier for this transition $\left(K_{s} \approx 1\right)$. This process induces a $2 \pi$ phase difference between the particle and the input signal. For larger noise, multiple transitions in one driving period are possible so that the phase difference would increase continuously with time and, as a practical matter, no phase synchronization can be observed. Since our interest is in the average synchronization time, only the noise range in which a single transition can possibly occur in one driving period is relevant. We thus have $P_{2 \pi}^{>}(D) \approx C_{1} \exp \left[-E_{d} / D\right] \exp \left[-E_{s} / D\right]$, where the constant $C_{1}$ is given by $C_{1}=\varepsilon \exp \left[\left(E_{d}+E_{s}\right) / D_{\text {opt }}\right]$. The average phase synchronization time for $D>D_{\text {opt }}$ is given by $\tau^{>}(D) \approx 1 / P_{2 \pi}^{>}(D)$. Taking the derivative we obtain

$$
\mathrm{d} \tau^{>}(D) /\left.\mathrm{d} D\right|_{D \rightarrow D_{o p t}^{+}} \approx-\frac{E_{d}+E_{s}}{\epsilon D_{o p t}^{2}} .
$$

Again, we observe that $\left|\mathrm{d} \tau^{>}(D) / \mathrm{d} D\right|_{D \rightarrow D_{o p t}^{+}} \rightarrow \infty$ for $\epsilon \rightarrow 0$. Equations (1) and (2) thus 


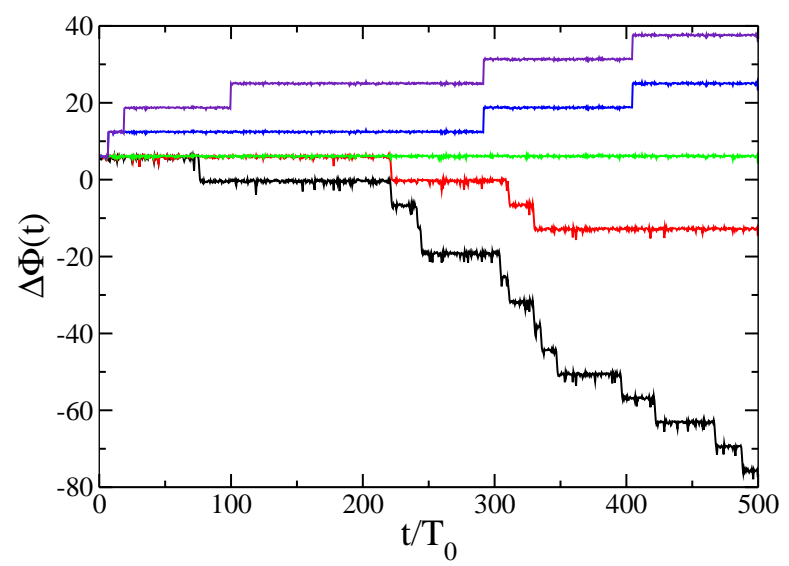

Fig. 3 - For the double-well potential system under periodic rectangular driving of amplitude $F_{0}=$ 0.18 , evolutions of the phase difference between the input and output for five different values of the noise amplitude.

indicate a cusp-like behavior in $\tau(D)$ about $D_{\text {opt }}$. Moreover, comparison between these two equations reveals that $\mathrm{d} \tau^{<}(D) /\left.\mathrm{d} D\right|_{D \rightarrow D_{o p t}^{-}} \neq\left|\mathrm{d} \tau^{>}(D) / \mathrm{d} D\right|_{D \rightarrow D_{o p t}^{+}}$. For small value of $\varepsilon$, for $D \rightarrow D_{o p t}^{-}$, the rise of $\tau^{<}(D)$ can be more pronounced than the rise of $\tau^{>}(D)$ for $D \rightarrow D_{o p t}^{+}$, or vice versa, depending on the system details. Thus, in general, we expect to see an asymmetric behavior in $\tau(D)$ near $D_{\text {opt }}$.

Notice that eqs. (1) and (2) were derived for regimes where $D<D_{\text {opt }}$ and $D>D_{\text {opt }}$, respectively. They only indicate a fast rising and a fast falling behavior in the average synchronization time for noise amplitude below and above the optimal value, respectively. Our argument is not applicable when the noise amplitude is in the infinitesimal vicinity of the optimal value. Thus, our heuristic theory cannot predict whether there is a cusp behavior in the mathematical sense of discontinuity in the derivative. Recent analytic expression $[16,17]$ for the instantaneous phase diffusion coefficient in a periodically driven system suggests, however, a smooth behavior in the phase-synchronization time about the optimal noise level. In particular, the diffusion coefficient shows a sharp but smooth peak at the optimal noise level. Since the average phase-synchronization time can be algebraically related to the diffusion coefficient [18], it is reasonable that the behavior of this time also be smooth.

We now present numerical evidence for the cusp-like behavior. Figure 3 shows, for the double-well potential system with periodic rectangular driving of amplitude $F_{0}=0.18$, evolutions of the phase difference between the input and output $x(t)$. Here the phase variable associated with the output is $\phi_{\text {out }}(t)=\tan ^{-1}[\bar{x}(t) / x(t)]$, where $\bar{x}(t)$ is the Hilbert transform of $x(t)$. The five traces shown from top down correspond to $D=0.037, D=0.035, D=0.03 \approx D_{\text {opt }}$, $D=0.025$, and $D=0.023$, respectively. Within the time considered (500 driving periods), we observe $2 \pi$ phase slips for all noise levels except for $D=0.03$, indicating that relatively long phase synchronization has been achieved and, hence, this is approximately the optimal noise amplitude. As $D$ deviates away from $D_{\text {opt }}, 2 \pi$ phase slips appear to occur more often.

To verify the cusp-like behavior in the average phase-synchronization time $\tau$, we choose a number of values of the noise amplitude about the optimal value. For each value, we use 20 realizations of the stochastic system to calculate the average value of the time between successive $2 \pi$ phase slips. The result is shown in fig. 4(a), where we see that $\tau$ exhibits apparently a cusplike behavior about the optimal noise amplitude $D_{\text {opt }}$. The asymmetric behavior in $\tau$ about 
(a)

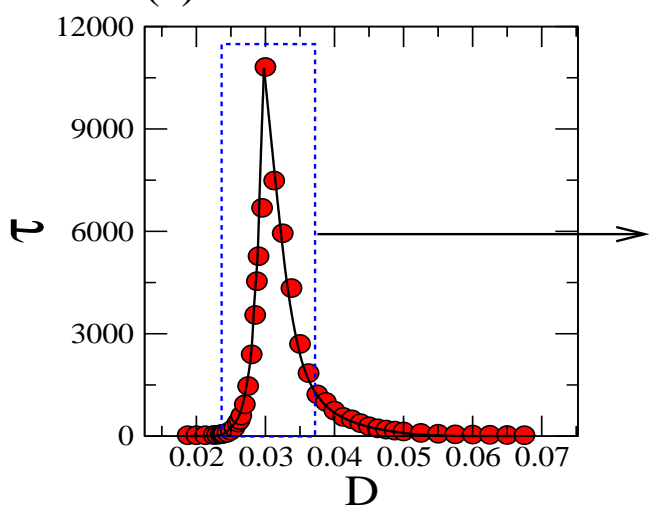

(b)

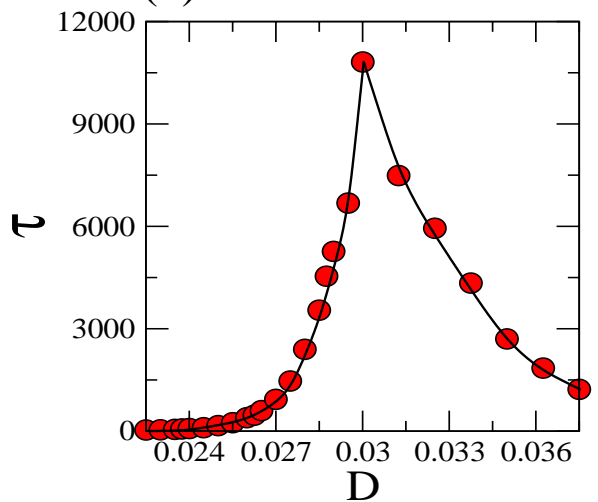

Fig. 4 - For the double-well potential system under periodic rectangular driving, (a) cusp-like behavior in the dependence of the average phase-synchronization time on noise amplitude, (b) asymmetric behavior of this dependence about the optimal noise amplitude.

$D_{\text {opt }}$, as predicted by our theoretical analysis, is shown in fig. 4(b), which is a blowup of part of fig. 4(a) near $D_{o p t}$. The behavior does not appear to depend on the form of the input signal, as we have verified using different choices of the input signals such as the sinusoidal signal.

In summary, we studied stochastic resonance by using the approach of phase synchronization and discovered that the average phase-synchronization time between the input and output signal in general exhibits an extremely high sensitivity to noise variation, characterized by a cusp-like behavior about the optimal noise amplitude. Numerical computations using different models and input signals as well as theoretical analysis suggest that the cusp-like behavior is general. While our analysis is heuristic, a more rigorous treatment may be possible using a recently proposed two-state, discrete phase model for SR [4]. Our finding can be potentially useful for applications such as device design and development based on stochastic resonance where a high degree of sensitivity to noise variation is required.

Our approach to understanding stochastic resonance may also be useful for the phenomenon of resonant activation [19] where, for a particle in a potential well with a timevarying barrier, in the presence of noise the average dwelling time can exhibit a minimum as a parameter controlling the barrier height varies. In previous works, the reported resonance peak is typically broad [19]. Our results here imply that if resonant activation is treated using phase synchronization, it is possible that the average synchronization time can exhibit a cusp-like, sharp maximum. A possible setting to establish this is to assume that the barrier height is controlled by a time-varying signal (e.g., chaotic) for which a phase variable can be defined. The relative phase difference between the particle and this signal, and consequently phase synchronization, can then be investigated as in this letter.

$$
* * *
$$

We thank Dr. A. NACHMAN for stimulating discussions about stochastic resonance and applications, which motivated the present work. We also thank Prof. P. HÄngGi, Prof. L. Schimansky-Geier, Prof. F. Marchesoni, Prof. M. Morillo, and Prof. J. M. Rubi for their insights and discussions. This work was supported by AFOSR under Grant No. F4962003-1-0290. 


\section{REFERENCES}

[1] Benzi R., Sutera A. and Vulpiani A., J. Phys. A, 14 (1981) L453; Benzi R., Parisi G., Sutera A. and Vulpiani A., J. Appl. Math., 43 (1983) 565.

[2] For review, see, for example: Wiesenfeld K. and Moss F., Nature (London), 373 (1995) 33; Gammaitoni L., Hänggi P., Jung P. and Marchesoni F., Rev. Mod. Phys., 70 (1998) 223; references there in.

[3] Shulgin B., Neiman A. and Anishchenko V., Phys. Rev. Lett., 75 (1995) 4157; Marchesoni F., Apostolico F. and Santucci S., Phys. Lett. A, 248 (1998) 332; Neiman A., Silchenko A., Anishchenko V. and Schimansky-Geier L., Phys. Rev. E, 58 (1998) 7118; Neiman A., Schimansky-Geier L., Moss F., Shulgin B. and Collins J. J., Phys. Rev. E, 60 (1999) 284; Anishchenko V., Moss F., Neiman A. and Schimansky-Geier L., Usp. Fiz. Nauk, 169 (1999) 7; Anishchenko V., Moss F., Neiman A. and Schimansky-Geier L., Sov. Phys. Usp., 42 (1999) 7; Neiman A., Pei X., Russell D., Wojtenek W., Wilkens L., Moss F., Braun H. A., Huber M. T. and Voigt V., Phys. Rev. Lett., 82 (1999) 660; Bahar S., Neiman A., Wilkens L. A. and Moss F., Phys. Rev. E, 65 (2002) 050901(R); Freund J. A., Schimansky-Geier L. and Hänggi P., Chaos, 13 (2003) 225.

[4] Freund J. A., Neiman A. B. and Schimansky-Geier L., Europhys. Lett., 50 (2000) 8; Rozenfeld R., Freund J. A., Neiman A. and Schimansky-Geier L., Phys. Rev. E, 64 (2001) 051107; Callenbach L., Hänggi P., Linz S. J., Freund J. A. and SchimanskyGeier L., Phys. Rev. E, 65 (2002) 051110; Lindner B., Garcia-Ojalvo J., Neiman A. and Schimansky-Geier L., Phys. Rep., 392 (2004) 321.

[5] Collins J. J., Chow C. C. and Imhoff T. T., Nature, 376 (1995) 236; Collins J. J., Chow C. C. and Imhoff T. T., Phys. Rev. E, 52 (1995) R3321; Collins J. J., Chow C. C., Capela A. C. and Imhoff T. T., Phys. Rev. E, 54 (1996) 5575; Gailey P. C., Neiman A., Collins J. J. and Moss F., Phys. Rev. Lett., 79 (1997) 4701.

[6] Inchiosa M. E., Bulsara A. R., Hibbs A. D. and Whitecotton B. R., Phys. Rev. Lett., 80 (1998) 1381; Hänggi P., Inchiosa M., Fogliatti D. and Bulsara A. R., Phys. Rev. E, 62 (2000) 6155; Stocks N. G., Phys. Rev. Lett., 84 (2000) 2310; Goychuk I., Phys. Rev. E, 64 (2001) 021909.

[7] Mcnamara B., Wiesenfeld K. and Roy R., Phys. Rev. Lett., 60 (1988) 2626.

[8] Wiesenfeld K., Pierson D., Pantazelou E., Dames C. and Moss F., Phys. Rev. Lett., 72 (1994) 2125.

[9] Longtin A., Bulsara A. and Moss F., Phys. Rev. Lett., 67 (1991) 656.

[10] Andó B. and Graziani S., IEEE Trans. Instrum. Meas., 52 (2003) 815.

[11] Longtin A. and Chialvo D. R., Phys. Rev. Lett., 81 (1998) 4012; Marino F., Giudici M., Barland S. and Balle S., Phys. Rev. Lett., 88 (2002) 040601.

[12] Park K., Lai Y.-C., Liu Z. and Nachman A., Phys. Lett. A, 326 (2004) 391.

[13] Rosenblum M. G., Pikovsky A. S. and Kurths J., Phys. Rev. Lett., 76 (1996) 1804.

[14] FitzHugh R. A., Biophys. J., 1 (1961) 445; Scott A. C., Rev. Mod. Phys., 47 (1975) 487.

[15] Kramers H. A., Physica, 7 (1940) 284; Hänggi P., Talkner P. and Borkovec M., Rev. Mod. Phys., 62 (1990) 251.

[16] Casado-Pascual J., Gómez-Ordóñez J., Morillo M., Lehmann J., Goychuk I. and Hängai P., Phys. Rev. E, 71 (2005) 011101.

[17] Prager T. and Schimansky-Geier L., cond-mat/0501078.

[18] Anishchenko V. S., Astakhov V. V., Neiman A. B., Vadivasova T. E. and SchimanskyGeIER L., Nonlinear Dynamics of Chaotic and Stochastic Systems. Tutorial and Modern Development (Springer, Berlin, Heidelberg) 2002.

[19] See, for example, Doering C. R. and Gadoua J. C., Phys. Rev. Lett., 69 (1992) 2318; Pechukas P. and Hänggi P., Phys. Rev. Lett., 73 (1994) 2772; Marchi M., Marchesoni F., Gammaitoni L., Menichella-Saetta E. and Santucci S., Phys. Rev. E, 54 (1996) 3479; Pankratov A. L. and Spagnolo B., Phys. Rev. Lett., 93 (2004) 177001. 